\title{
Appendage patterning in the South American bird spider Acanthoscurria geniculata (Araneae: Mygalomorphae)
}

\author{
Matthias Pechmann • Nikola-Michael Prpic
}

Received: 4 December 2008 / Accepted: 19 February 2009/Published online: 6 March 2009

(C) The Author(s) 2009. This article is published with open access at Springerlink.com

\begin{abstract}
Pattern formation by the genes dachshund (dac), Distal-less (Dll), extradenticle (exd) and homothorax (hth) in spider appendages has been studied previously only in members of the higher spiders (Araneomorphae). In order to study the diversity and conservation of pattern formation in spiders as a whole, we studied homologs of these genes in embryos of the bird spider Acanthoscurria geniculata, which belongs to the Mygalomorphae, a more primitive spider group. We show that the patterns of $d a c$ and $D l l$ are largely conserved in all spiders studied so far. We find a duplication of $h t h$ and exd genes as previously identified in the higher spider Cupiennius salei. These data suggest that pattern formation shows little diversity in all spiders, including the duplication of $h t h$ and exd that likely occurred before the split of Mygalomorphae and Araneomorphae. We also find that the legs and pedipalps bear endites of which only the pedipalpal endite expresses $D l l$ and is retained in the adult. Similarly, the limb buds of the posterior spinnerets express $\mathrm{Dll}$ and become segmented appendages in the adult, whereas the anterior spinnerets lack $D l l$ expression and are absent in postembryonic stages. In both cases, the expression of $D l l$ or the lack of it indicates structures which will be retained as adult traits or rudimentary structures that degenerate, respectively. The presence of embryonic rudiments of leg endites in Acanthoscurria and the leg-like pattern formation in the
\end{abstract}

Communicated by S. Roth

M. Pechmann • N.-M. Prpic $(\bowtie)$

Johann-Friedrich-Blumenbach-Institut für Zoologie

und Anthropologie, Abteilung Entwicklungsbiologie,

GZMB Ernst-Caspari-Haus, Georg-August-Universität,

Justus-von-Liebig-Weg 11,

37077 Göttingen, Germany

e-mail: nprpic@uni-goettingen.de posterior spinnerets are interpreted as primitive traits that have been lost in the Araneomorphae.

Keywords Spiders · Appendage development .

Patterning genes $\cdot$ Rudiments $\cdot$ Spinnerets

\section{Introduction}

The evolutionary success of the arthropods is, to a large part, founded on the diversity of their appendages which have been adapted to a large number of functions. A major goal of evolutionary developmental biology is to understand how evolutionary changes in developmental genetic mechanisms lead to novel morphological traits, e.g. new appendage types, that can adapt to new functions.

Spiders have a number of different appendage types along their body axis. The opisthosoma (abdomen) bears four pairs of highly specialised appendages: the appendages on opisthosomal segments 2 and 3, after a short phase of outgrowth, invaginate and give rise to a complex respiratory system inside of the body. The appendages on opisthosomal segments 4 and 5 are the spinnerets that form a silk spinning and weaving apparatus. The appendages on the prosoma (head and trunk) are mainly used for feeding and locomotion, but can have additional functions as well. The most anterior appendage is the bilobed labrum that likely evolved from an anterior appendage pair by rotation and fusion (Kimm and Prpic 2006) and that serves as the upper lip during feeding. The following pair of appendages, the chelicerae, are used for prey capture and feeding and they inject the venom into prey animals. The next pair, the pedipalps, are a multifunctional appendage pair, which is used for sensory perception, feeding and, in males, sperm transfer during mating. The following four pairs of walking 
legs are mainly locomotory appendages, but are also equipped with organs for sensory perception and are also used for other functions such as prey capture.

Previous studies in spiders have shown that the patterning of pedipalps and legs is very similar (Abzhanov and Kaufman 2000; Prpic et al. 2003; Prpic and Damen 2004), probably reflecting their morphological similarity. Pattern formation in the chelicera, however, is different from the legs and pedipalps, suggesting that the differences in pattern formation correlate with the specific morphology of the chelicera (Prpic and Damen 2004). However, these studies have only involved representatives of the higher spiders, the Araneomorphae (for an overview of spider phylogeny, see Fig. 1a). Members of the two other spider groups, the Mesothelae and the Mygalomorphae, have not yet been studied. Thus, it is currently unclear whether the patterns in the araneomorph members studied so far are representative of the spiders as a whole. The Mesothelae (less than 100 described species) are a basally branching spider group and display a number of primitive traits including a fully segmented opisthosoma (hence, their common name "segmented spiders"). Unfortunately, these spiders are rare, very difficult to breed and embryos are not yet available for molecular studies. We have, therefore, used a member of the spider group branching after the Mesothelae, the Mygalomorphae (bird spiders). The studied species, Acanthoscurria geniculata (Fig. 1d), is a large and colourful bird spider distributed in Brazil (South America). We have studied the expression of several leg patterning genes in Acanthoscurria embryos and we show that the patterns are largely conserved between mygalomorph spiders and the higher spiders of the Araneomorphae. However, A. geniculata also shows traits like embryonic endite rudiments on the legs and leg-like morphology and pattern formation in the posterior spinnerets, which we interpret as primitive characters lost in the Araneomorphae.

\section{Materials and methods}

Embryo collection and fixation

Embryos of A. geniculata were obtained from a female in the private collection of the first author. Only a single cocoon was available for study. The embryos of Cupiennius salei and Achaearanea tepidariorum were obtained from our laboratory stocks in Göttingen. Embryos of all three species were fixed according to the published protocol for Cupiennius embryos (Prpic et al. 2008a).

\section{Gene cloning}

Total RNA was isolated using Trizol (Invitrogen) according to the manufacturer's instructions and cDNA was synthesised
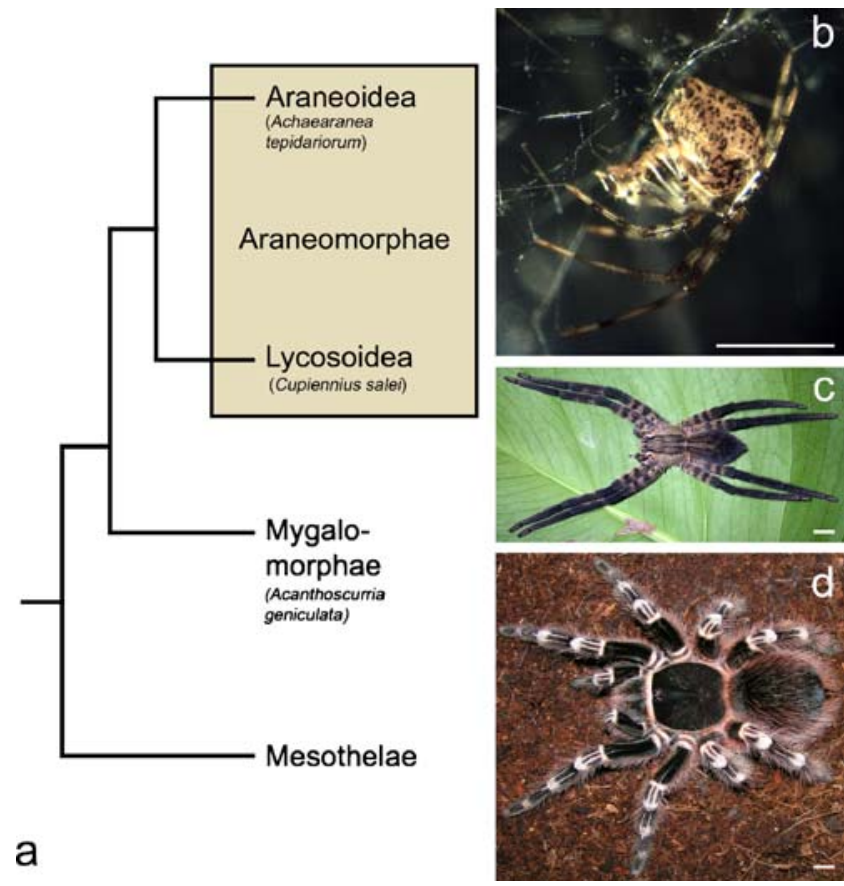

a

Fig. 1 Overview of the phylogenetic relationships of the spider species used in this work. a Simplified phylogenetic tree showing the relationships of the three major spider groups: Mesothelae, Mygalomorphae, and the higher spiders Araneomorphae (boxed). The names of the species in this study are given in parentheses below the names of the larger taxa. Photos of adult females of the species in this study: b A. tepidariorum, c C. salei and $\mathbf{d} A$. geniculata. Scale bar is $1 \mathrm{~cm}$ in all panels

with the Smart polymerase chain reaction (PCR) cDNA Synthesis Kit (Clontech). Fragments of the genes Dll, dac, exd and hth were isolated by PCR using the previously published primers (Prpic et al. 2001, 2003; Prpic and Tautz 2003). The $h t h-2$ fragment, however, resulted from priming of the nested reverse primer only. This is the reason why it is shorter than the hth-1 fragment which resulted, as expected, from the priming of the nested forward and reverse primer pair. The orthology of all cloned fragments was assessed by phylogenetic analysis as described previously (Prpic et al. 2005). The sequences of the fragments are available in GenBank under the following accesion numbers: Acanthoscurria: Dll (FM876228), dac (FM876227), hth-1 (FM876231), hth-2 (FM876232), exd-1 (FM876229), exd-2 (FM876230); Cupiennius: Dll (AJ278606); Achaearanea: Dll (FM876233).

In situ hybridisation and nuclear stains

The detection of mRNA in fixed embryos has been performed by whole-mount in situ hybridisation (Prpic et al. 2008b). For DNA staining, fixed embryos were rehydrated stepwise in phosphate-buffered saline with 0.1 $\%$ Tween-20 (PBST) and incubated for $1 \mathrm{~h}$ in Sytox Green 
(1:5,000 in PBST). Incubation was followed by several washes in PBST. Retained Sytox Green was visualised with UV light under a Leica dissection microscope equipped with an Intas digital camera. Spider appendages were dissected as described previously (Prpic et al. 2008c) and images were captured with a Zeiss Axioplan-2 microscope equipped with an Intas digital camera. All digital images have been subjected to adjustment of brightness, colour values and contrast using Adobe Photoshop 7.0 for Apple Macintosh.

\section{Results}

\section{External morphology of Acanthoscurria geniculata embryos}

We have examined embryos of $A$. geniculata at the late inversion stage. The embryos are huge compared to the embryos of $C$. salei and A. tepidariorum, two other spider species commonly used in comparative developmental studies (for an image of the adult animals, see Fig. 1b, c; McGregor et al. 2008). The embryos (approximately $2 \mathrm{~mm}$ in diameter) are more than double the size of Cupiennius embryos and more than four times the size of Achaearanea embryos (Fig. 2a). At the late inversion stage, the germ band is split along the ventral midline and dorsal closure is almost complete. At the anterior end, the ectoderm has almost fully overgrown the brain anlage (for details on spider neurogenesis, see Stollewerk 2002) and future anterior dorsal tissue is growing from the anterior rim of the head lobes (Fig. 2b). In lateral view, it can be seen that a large portion of the dorsal tissue is already present (Fig. 2c).

At the anterior end, directly behind the brain, is a bilobed labrum, followed by a pair of chelicerae (Fig. 2b). The chelicerae comprise a proximal base and a slightly tapered distal end that we term the "crown" and that will give rise to the claw in the fully developed appendage. The following appendages of the prosoma are one pair of pedipalps (Fig. 2b) and four pairs of walking legs (Fig. 2c). In Acanthoscurria, the embryonic pedipalps and walking legs are morphologically very similar. The pedipalp is almost as long as the walking legs (Fig. 3b). Intriguingly, all legs and the pedipalp have a proximal protrusion which is the primordium of the gnathendite (Fig. 3a, b). This is surprising because, in the adult animals, only the pedipalp has a large gnathendite whereas the walking legs do not have gnathendites. However, the gnathendite growing from the embryonic pedipalp is larger than the gnathendites on the walking legs, probably already reflecting the beginning reduction of the walking leg gnathendites.

The opisthosoma has four pairs of appendages (Fig. 3c). The first two pairs are morphologically virtually identical at
Fig. 2 Morphology of Acanthoscurria embryos at the late inversion stage. All embryos are stained with Sytox Green. a Size comparison of late inversion stage embryos of $A$. geniculata, C. salei and A. tepidariorum. All embryos are to scale (scale bar is $500 \mu \mathrm{m}$ ) and anterior is up. b Frontal view of an Acanthoscurria embryo. The brain $(b r)$ and the appendages (see abbreviations below) are labelled. The arrow denotes dorsally growing tissue. c Lateral view of an Acanthoscurria embryo. The appendages and the opisthosomal segments are labelled (see abbreviations below). The arrow points to the invagination sites in the ventral nervous system of the opisthosoma. $b r$ brain, $c h$ chelicera, $L 1-L 4$ walking leg 1 to $4, l r$ labrum, O1-O5 opisthosomal segment 1 to 5 , $p p$ pedipalp
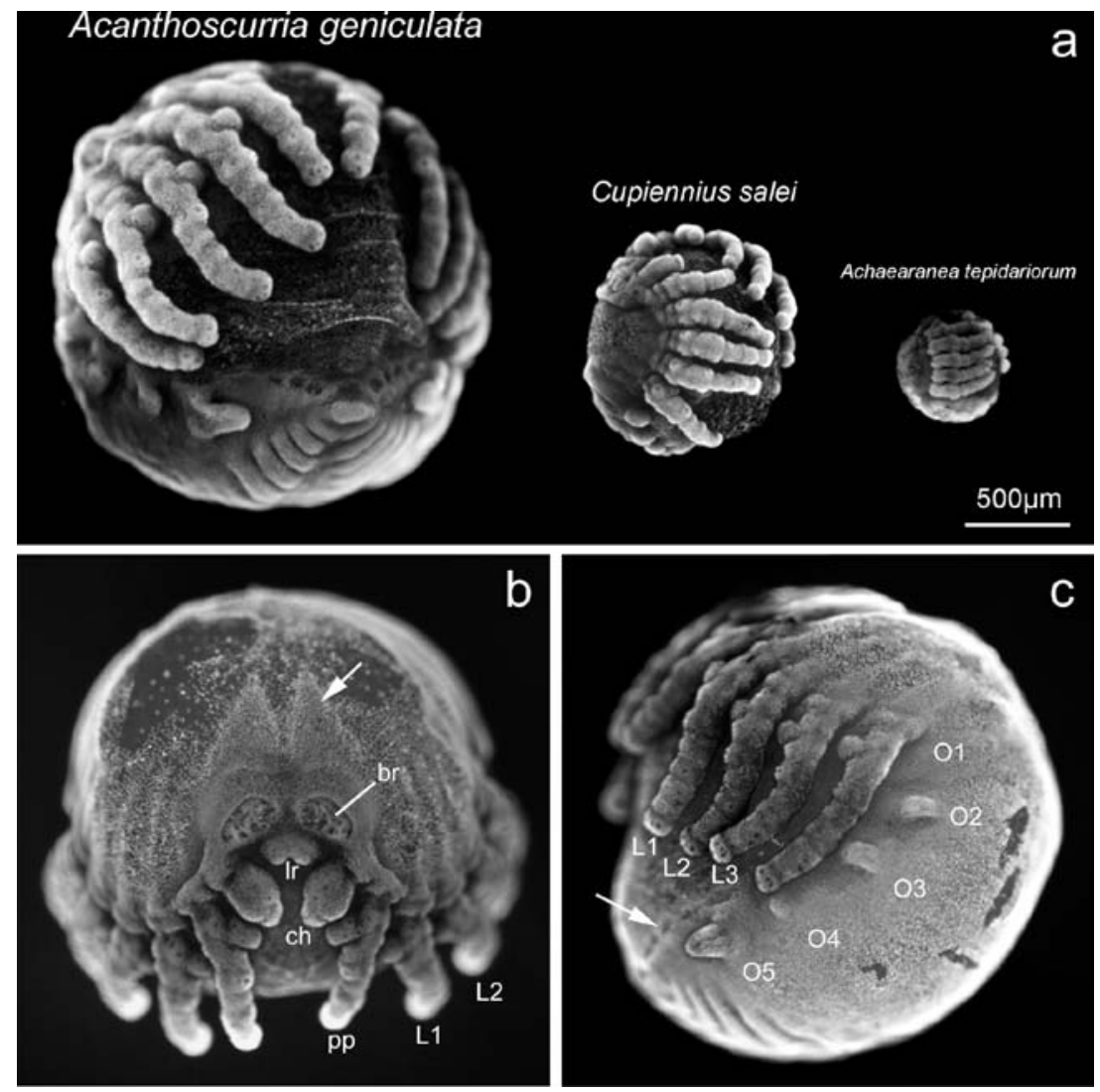

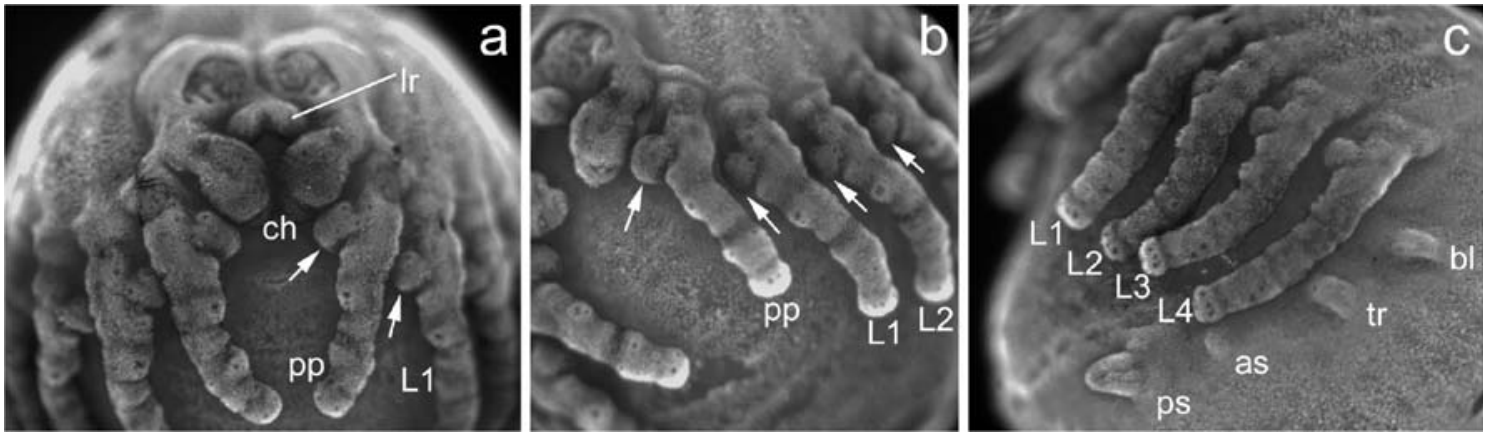

Fig. 3 Morphology of the Acanthoscurria embryonic appendages. All embryos are stained with Sytox Green. a Frontal view. Note the bilobed labrum $(l r)$ right behind the brain anlage. The arrows point to endites on the pedipalp and legs. b Lateral view of the prosoma, anterior to the left. The arrows point to endites on the pedipalp and

this stage and will give rise to the breathing organs. There is a small pit behind these appendage buds, which we interpret as the beginning invaginations of the future respiratory system. The next pair of opisthosomal appendages is very small. These limb buds are the anlage of the anterior pair of spinnerets. The anterior pair of spinnerets is present in many spider groups, but is completely reduced in adult mygalomorph spiders. The following appendage pair is significantly larger and will give rise to the posterior pair of spinnerets.

Isolation of leg patterning genes from Acanthoscurria geniculata

In order to study pattern formation in these appendages, we have isolated a set of appendage patterning genes that have already been used for this purpose in a number of other

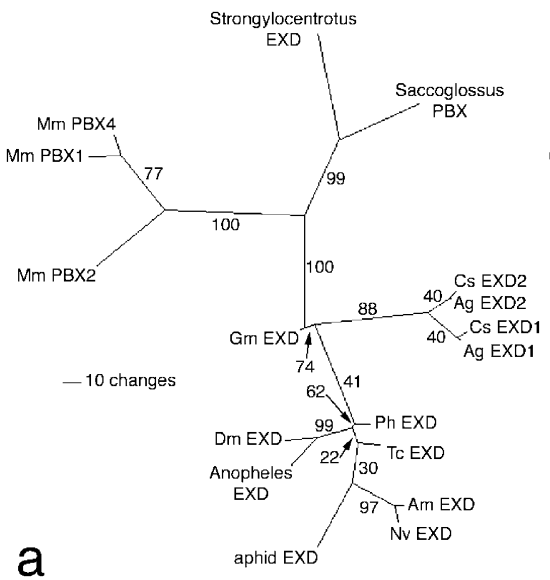

Fig. 4 Phylogenetic analysis of the exd and hth sequences from $A$. geniculata. Shown is the 50\% majority-rule consensus tree for exd (a) and hth (b). The search method was full heuristic and the optimality criterion was set to parsimony. The numbers on the edges are bootstrap resampling values. Species abbreviations: $A g A$. geniculata, Am Apis mellifera, Cs C. salei, Dm Drosophila melanogaster, Gb legs. c Lateral view of the opisthosoma, anterior to the left. Abbreviations see Fig. 2. Additional abbreviations: $b l$ book lung primordium, $t r$ trachea primordium, as anterior spinneret rudiment, $p s$ posterior spinneret primordium

arthropod species, including spiders (e.g. Abzhanov and Kaufman 2000; Prpic et al. 2003). A fragment of the homeobox of the Acanthoscurria Dll gene was isolated using the previously published degenerate $\mathrm{Dll}$ homeobox primers (see the Materials and methods section). The sequence of this fragment was then used to design nested gene-specific primers that were used together with the previously published dlx motif 1 primer (Prpic and Tautz, 2003) to amplify a larger portion of the Acanthoscurria Dll gene. While only a single dac gene fragment could be isolated, the cloning of the exd and hth fragments yielded two different fragments for each gene. Phylogenetic analysis of these fragments (Fig. 4a, b) revealed that they are orthologous to genes known from another spider, C. salei, and we designate the Acanthoscurria genes from which the fragments derive accordingly as exd-1, exd-2, hth-1 and hth2. These data suggest that the duplication of the $h t h$ and exd

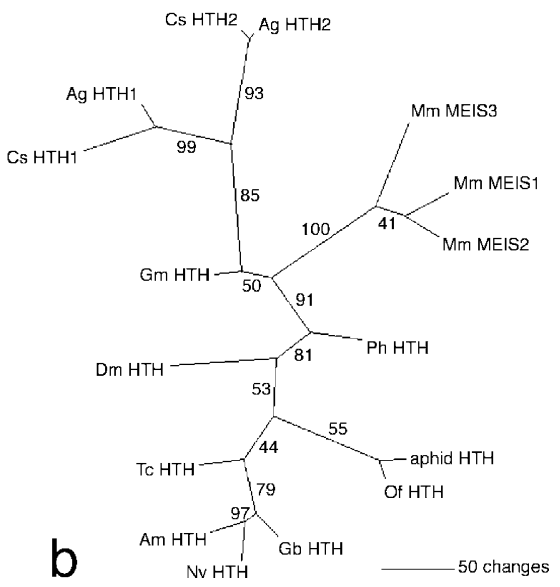

Gryllus bimaculatus, Gm G. marginata, Mm Mus musculus, Nv Nasonia vitripennis, Of Oncopeltus fasciatus, Ph P. hawaiensis, Tc Tribolium castaneum. Anopheles denotes A. gambiae; Saccoglossus denotes S. kowalevskii; Strongylocentrotus denotes S. purpuratus; aphid denotes Acyrthosiphon pisum 
genes occurred at the base of Araneae before the split between Mygalomorphae and Araneomorphae.

Embryonic expression of leg patterning genes

We next determined the expression patterns of these genes by whole-mount in situ hybridisation. We report first on the expression in the whole embryo and focus on the pattern in the appendages in the next chapter.

Apart from the appendages, $d a c$ is strongly expressed in the central nervous system (Fig. 5b, c). The strongest expression is detected in the celiceral neuromere (Fig. 5c), and a slightly weaker expression is seen in groups of cells in the protocerebrum (Fig. 5c) along the anterior rim of the head lobes and in the ventral nerve cord. Expression of $d a c$ is also detected in the ectodermal regions of the hind gut, the stomodaeum. In the forming heart, strong expression of $d a c$ is detected (Fig. 5a).

The $D l l$ gene is mainly expressed in the appendages (see below). However, there is a weak expression domain in a group of cells in the head lobes (Fig. 5f) and there is also a small expression domain at the posterior end of the germ band (Fig. 5d, e).

The two exd genes are expressed at a low level in the head lobes (Fig. 6b, d). In addition, exd-2 is expressed in the dorsal tissue of the opisthosoma in a segmental fashion (Fig. 6c), indicating the presence of metameric units in this area. A similar expression pattern is seen with $h t h-1$ and $h t h-2$ (Fig. 6e, g), but the dorsal stripes of $h t h-1$ expression are thinner and more defined than the exd-2 and $h t h-2$ stripes. The genes exd-2 and $h t h-1$ are also expressed in the head lobes and in the ventral nerve cord (Fig. 6d, f).

Fig. 5 Embryonic expression of dac and Dll in Acanthoscurria. a-c Expression of dac. $\mathbf{d}-\mathbf{f}$ Expression of Dll. a, d Embryo in lateral view. $\mathbf{b}$, e Embryo in frontal view. c, f Magnified view of the anterior end of the embryo. The arrow in $\mathbf{e}$ and $\mathbf{f}$ denotes faint expression of $\mathrm{Dll}$ in the head lobes. Abbreviations see Figs. 2 and 3. Additional abbreviations: $c n$ cheliceral neuromere, $h$ heart, $h g$ hind gut, $h l$ head lobe, $p c$ protocerebral expression domains, pe posterior end of the germ band, vnc future ventral nerve cord
Expression of $d a c, D l l$, exd and $h t h$ in the appendages

The bilobed labrum strongly expresses $\mathrm{Dll}$ and the expression is strongest at the two tips of the labrum (Fig. 5f). We could not detect expression of $d a c$ in the labrum (Fig. 5c, and data not shown). Both exd and hth genes are expressed in the labrum. The expression of exd-1, exd-2 and hth-1 in the labrum is relatively strong (Fig. 6b, d, f), whereas $h$ th -2 is expressed at a low level (Fig. 6h).

The chelicera does not express dac (Fig. 7a). The expression of $\mathrm{Dll}$ fills almost the entire chelicera except for the most proximal portion (Fig. 7b). Both hth-1 and exd1 are expressed throughout the chelicera (Fig. 7c, e), whereas $h t h-2$ is expressed in a weak medial ring and in the crown (Fig. $7 \mathrm{~d}$ ), and exd-2 is expressed in the proximal two thirds of the chelicera with a straight and sharp border of expression near the distal end of the basal part of the chelicera (Fig. 7f).

The legs and the pedipalp all show very similar expression patterns of all genes. The dac gene is expressed in a medial domain that corresponds to the future segments trochanter and femur in both appendage types (Fig. $7 \mathrm{~g}, \mathrm{~m}$ ). $\mathrm{Dll}$ is expressed in the distal portion of pedipalps and legs (Fig. $7 \mathrm{~h}, \mathrm{n}$ ) that will give rise to the future leg segments femur, patella, metatarsus and tarsus and the future pedipalp segments femur, patella and tarsus. There is an additional expression domain in the tip of the gnathendite of the pedipalp (Fig. 7h), whereas the smaller gnathendites of the walking legs do not express $D l l$ (Fig. 7n). The $h t h-1$ gene is expressed throughout the leg and pedipalp except for the tarsal segment (Fig. 7i, o). The hth-2 gene is expressed in segmental rings in both pedipalp and leg (Fig. $7 \mathrm{j}, \mathrm{p}$ ). The
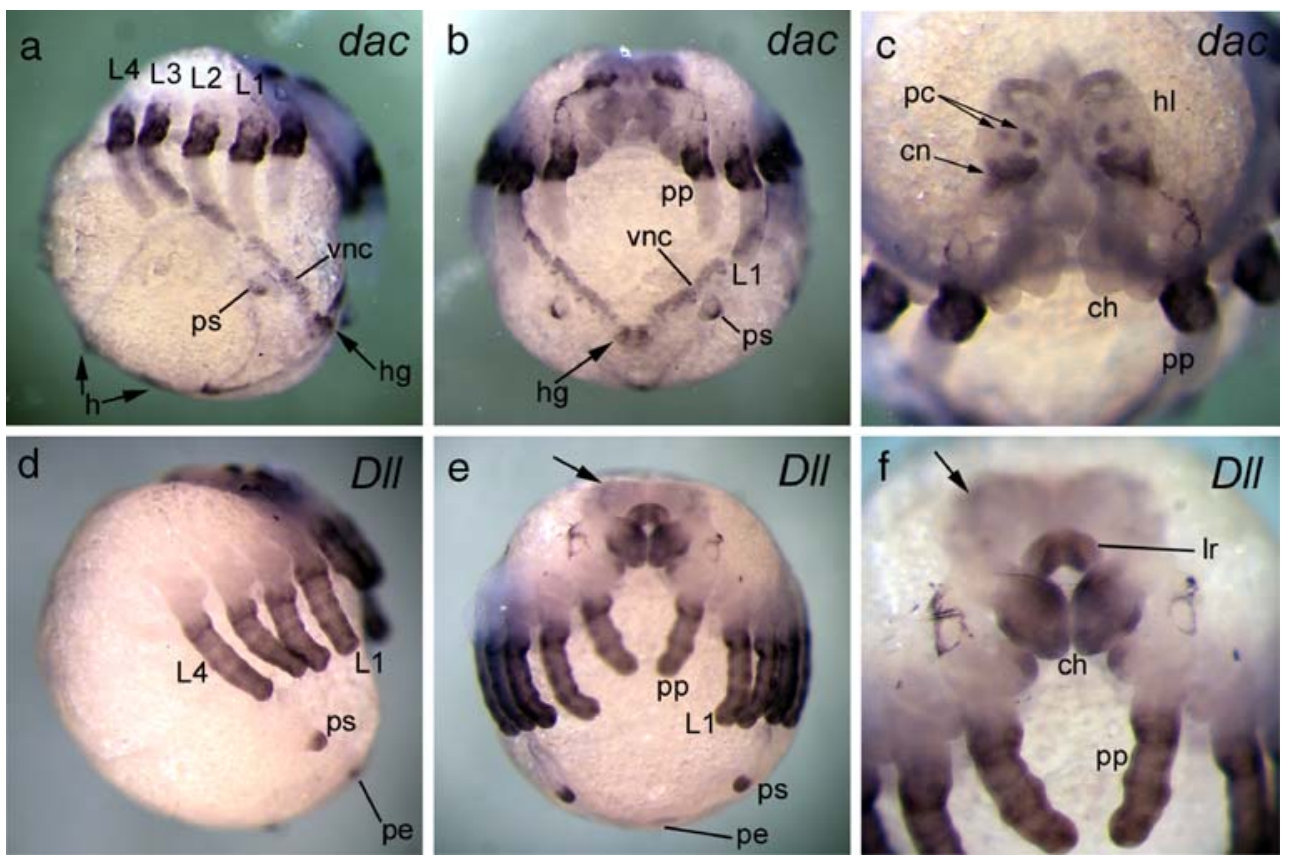

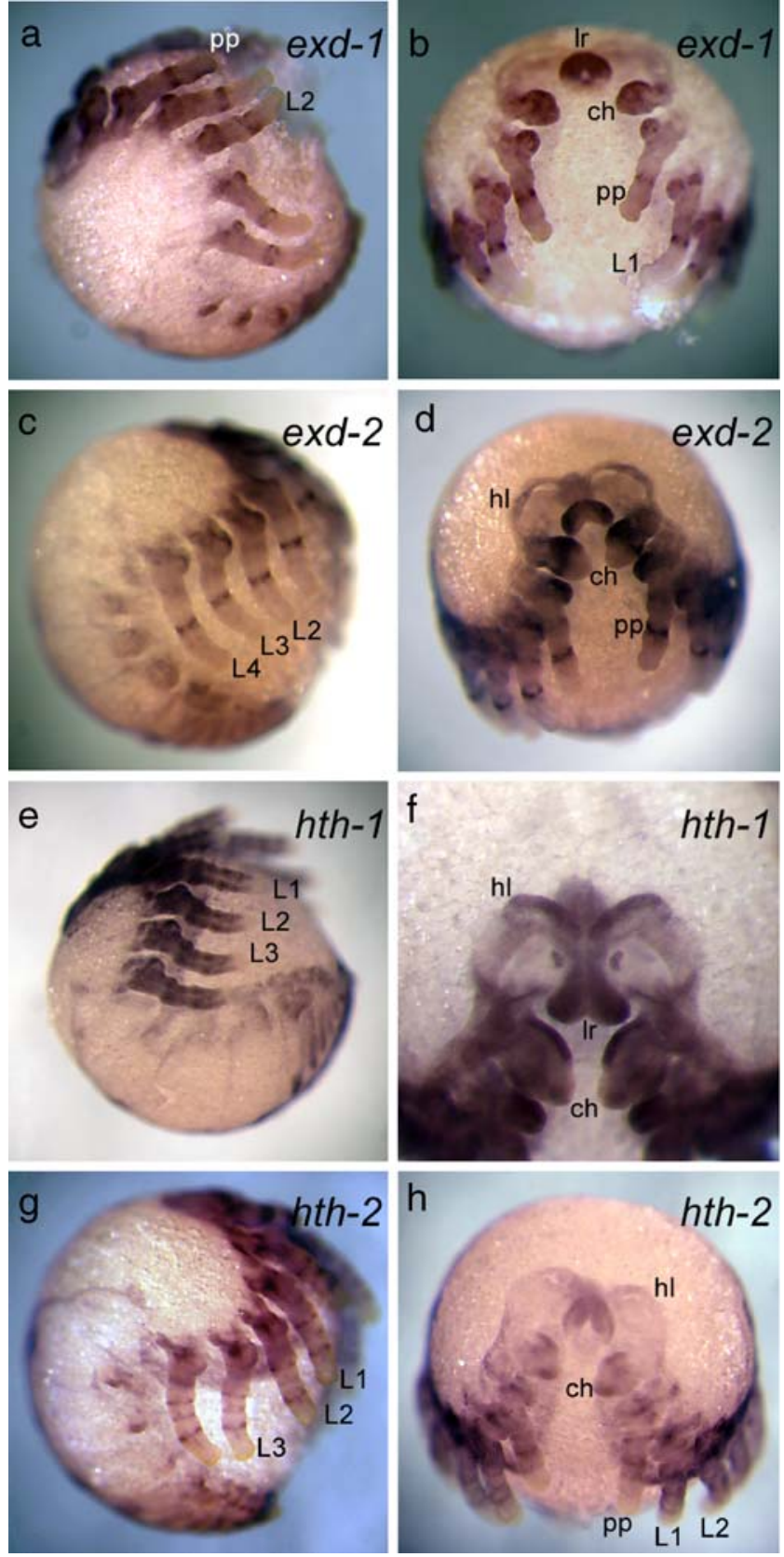

Fig. 6 Embryonic expression of exd and hth genes in Acanthoscurria. a, b Expression of exd-1. c, $\mathbf{d}$ Expression of exd-2. e, $\mathbf{f}$ Expression of $h t h-1$. g, h Expression of $h t h-2$. a, c, e, g Embryos in lateral view. b, $\mathbf{d}, \mathbf{f}, \mathbf{h}$ Embryos in frontal view. Abbreviations see Figs. 2 and 3. Additional abbreviation: $h l$ head lobe

rings of expression coincide with the future borders between trochanter and femur, femur and patella, patella and tibia and tibia and the next segment, which is the tarsus in the pedipalp and the metatarsus in the leg. There is also a cloudy expression in the coxa of both appendage types. The expression patterns of exd-1 and exd-2 are very similar (Fig. 7k, 1, q, r). There is a diffuse expression in the coxa, a stronger expression domain in the gnathendite and a ring at the future border between patella and tibia. In the exd-2 pattern, there is an additional, very weak ring of expression at the future border between trochanter and femur.

The genes exd-1, exd-2 and hth-2 are expressed in all four opisthosomal appendages (Fig. 6a, c, g), whereas dac and $\mathrm{Dll}$ are only expressed in the posterior spinnerets (Fig. 5a, b, d, e). The posterior spinnerets of adult Acanthoscurria are leg-like and consist of three movable segments. In order to compare the expression patterns in the legs with those in the posterior spinnerets, we have analysed the expression patterns in the posterior spinnerets in more detail. The dac gene is expressed in a medial domain in the posterior spinneret bud, but this ring is incomplete on the ventral side (Fig. 7s). Dll is expressed in the distal half of the posterior spinnerets (Fig. 7t). The hth-1 gene is expressed throughout the posterior spinneret, but there is a clearing of the expression in the tip (Fig. $7 \mathrm{u}$ ) that is similar to the complete absence of expression in the tarsus of pedipalp and leg (Fig. 7i, o). The genes $h t h-2$, exd1 and exd-2 are expressed at the base of the posterior spinnerets (Fig. 7v, w, x).

\section{Discussion}

Patterning of the prosomal appendages is very similar to higher spiders

The set of genes studied here has already been studied in detail in the prosomal appendages of a higher spider species, the ctenid C. salei (Prpic et al. 2003; Prpic and Damen 2004). A limited set of data is also available for the spider Steatoda triangulosa (Abzhanov and Kaufman 2000). The available data show that the expression of dac and $\mathrm{Dll}$ in the prosomal appendages is virtually identical in all three spider species. This suggests that, at the level of these patterning genes, there is no significant diversity of patterning mechanisms in the entire Arachnida. In Cupiennius, some additional expression domains of $d a c$ in the proximal coxa of the pedipalp and legs and a late basal expression within the chelicera have been reported (Prpic and Damen 2004). These expression domains are lacking in Acanthoscurria; however, the extra domains in Cupiennius are only present during some developmental stages and might have been missed in the limited material of Acanthoscurria that we had available for study.

The genes exd and hth are present in two paralogs each in Cupiennius (Prpic et al. 2003), and the same situation is present in Acanthoscurria. The proteins encoded by the two genes are known co-factors in Drosophila and are involved in providing proximal positional information during $\mathrm{PD}$ axis formation (e.g. Abu-Shaar and Mann 1998). It has been argued previously that one set of paralogs, namely, 


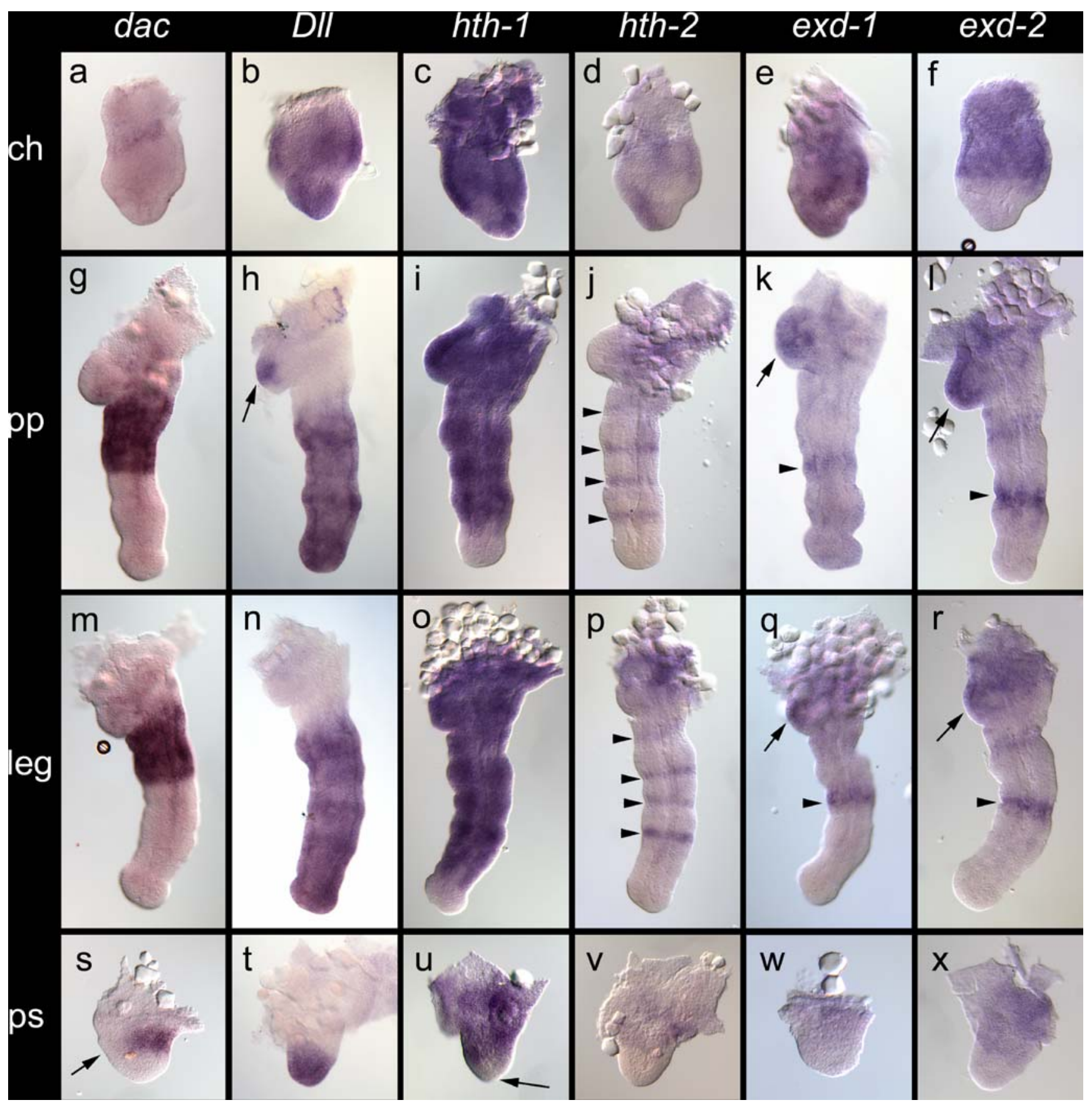

Fig. 7 Expression of patterning genes in the appendages of Acanthoscurria. a-f Expression patterns in the chelicera. $\mathbf{g}-\mathbf{l}$ Expression patterns in the pedipalp. $\mathbf{m}-\mathbf{r}$ Expression patterns in the leg. $\mathbf{s}-\mathbf{x}$ Expression patterns in the posterior spinneret. The appendages are arranged in columns according to the gene and the gene name is given above the columns. The arrows in $\mathbf{h}, \mathbf{k}, \mathbf{l}, \mathbf{q}, \mathbf{r}$ point to the expression

hth-1 and exd-1, provides the same function in spiders (Prpic et al. 2003). The other set, hth-2 and exd-2, based on its expression in segmental rings, is likely involved in leg segmentation. Indeed, recent work has shown that the distal ring of the exd-1 pattern in Cupiennius is activated by the Notch pathway which is also involved in leg segmentation in the embryonic gnathendites. The arrowheads in $\mathbf{j}-\mathbf{l}, \mathbf{p}-\mathbf{r}$ point to the segmental rings of expression. The arrow in $\mathbf{s}$ points to the incomplete ring of dac expression. The arrow in $\mathbf{u}$ points to the clearing at the tip in the $h t h-1$ pattern. Proximal is up and ventral is to the left in all panels. Abbreviations see Figs. 2 and 3

(Prpic and Damen 2009). In Acanthoscurria, both exd genes are expressed in the proximal leg and in distal rings and thus could both combine a proximal and a segmental role. The two Acanthoscurria hth genes are expressed like their Cupiennius homologs, except that the $h t h-1$ ring in the tarsus and the distal-most $h t h-2$ ring at the metatarsal/tarsal 
joint are missing. The Acanthoscurria patterns are consistent with a role of $h t h-1$ in PD axis patterning and $h t h-2$ in leg segmentation. It has been noted previously that the expression patterns of exd-1 and hth-l are reversed compared to the expression of exd and hth in Drosophila and other insects (Prpic et al. 2003). The Acanthoscurria data provide further evidence that the reversed condition is common to all spiders. The reversed condition is also found in the myriapod Glomeris marginata (Prpic and Tautz 2003), whereas the crustacean Parhyale hawaiensis shows a situation similar to insects, and it has been proposed before that the reversal of the exd and hth expression patterns occurred in the pancrustacean lineage (Prpic and Telford 2008). It is unclear, however, whether the reversed condition shared by spiders and myriapods is a synapomorphy of the two groups or rather represents a plesiomorphic state tracing from an arthropod ancestor.

In the antenna of Drosophila, exd and hth overlap throughout the appendage which has been shown to be a prerequisite for the development of antennal morphology (Dong et al. 2000, 2002). It has previously been noted that the expression patterns of $D l l, d a c, e x d-1$ and $h t h-1$ in the chelicera resemble those in the antenna of Drosophila (Prpic and Damen 2004). The insect antenna is homologous to the spider chelicera (Damen et al. 1998; Telford and Thomas 1998; Mittmann and Scholtz 2003), suggesting that the similarities indicate a common homologous patterning process of the arthropod antennal appendage. However, the status in outgroups is not known and the Drosophila condition is not conserved in the antennae of other insects (Gryllus, Tribolium) (Ronco et al. 2008; Toegel et al. 2009). This supports the alternative hypothesis that the pattern similarities in spiders and Drosophila are homoplasies.

Gnathendites are in the ground-plan of the arachnid post-cheliceral appendage

Ventral outgrowths from the main appendage axis, termed endites, are present in all arthropod groups, including extinct fossil groups (e.g. Ramsköld and Edgecombe 1996). In insects, for instance, two endites each are present on the maxilla and the labium, and crustacean appendages can have multiple endites along the ventral side of the appendages. The most proximal endite on an appendage is called the gnathendite because, in most cases, it is used for food processing and thus has a gnathal function.

Spiders do not possess gnathendites on the walking legs and the chelicera, but a large gnathendite is present on the coxa of the pedipalp. Intriguingly, Acanthoscurria embryos show endites growing from the coxae of the walking legs as well. These are not retained in the adult and thus are embryonic rudiments. The endite on the pedipalp expresses $D l l$; the lack of $D l l$ expression in the endites on the walking legs might be correlated with the later degeneration of these structures.

The presence of rudimentary gnathendites on the walking legs argues against the notion that the gnathendite is a specific innovation of the pedipalpal appendage and strongly suggests that the presence of a fully developed gnathendite is in the ground pattern of the arachnid postcheliceral appendage. The homology of this gnathendite and the gnathendites in other arthropod groups is currently unclear. Developmental genetic data, including the proximal co-expression of exd and hth (e.g. Prpic et al. 2003) and the function of the Dll gene (e.g. Beermann et al. 2001; Schoppmeier and Damen 2001; Khila and Grbic 2007), argue for the homology of the proximal part of the legsthe coxa plus the gnathendite-in all arthropods. Fossil data, however, suggest that the chelicerate coxa is homologous to the crustacean basis; the crustacean coxa is thought to be derived from a proximal endite in an ancestral appendage type (Waloßek 1995). The arachnid gnathendite could be homologous to this proximal endite and, by inference, the crustacean coxa. The arachnid post-cheliceral appendages would thus represent a primitive appendage type before the evolution of a "true" coxa. Further studies involving additional proximal genes, e.g. teashirt, might resolve this issue further.

Distal-less expression prefigures the number of adult spinnerets

The Mesothelae (segmented spiders) and the Araneomorphae (higher spiders) have two pairs of spinnerets in the adult: one anterior pair and one posterior pair in opisthosomal segments 4 and 5 , respectively. In addition, there are sometimes so-called median spinnerets (Fig. 8g, h). These, however, are secondary outgrowths formed by splitting from the posterior spinnerets (Foelix 1996). The third group of spiders, the Mygalomorphae, to which Acanthoscurria belongs, lack the anterior pair of spinnerets. However, embryonic rudiments of these spinnerets are present (see Fig. 3c). The posterior pair in the adults is strikingly leglike and has three segments (Fig. 8i). This morphology and the leg-like gene expression patterns shown in this work suggest that the spinnerets are serially homologous to legs. In contrast to this notion, previous work has argued that spinnerets are homologous to the gills of crustaceans and the wings of insects (Damen et al. 2002). Our data do not support this hypothesis; however, more data, especially the expression of wing- or gill-specific genes, are required to further resolve this issue.

Interestingly, the embryonic rudiments of the anterior spinnerets in Acanthoscurria do not express Dll while the posterior spinnerets express $D l l$ strongly (see Fig. 8c, d). Similar to the gnathendites in the pedipalp and the walking 


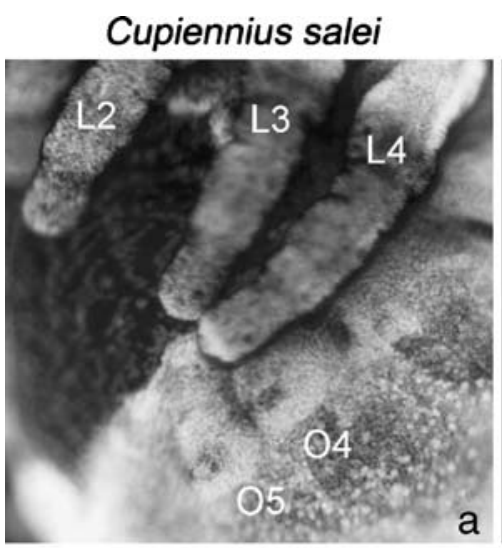

Achaearanea tepidariorum
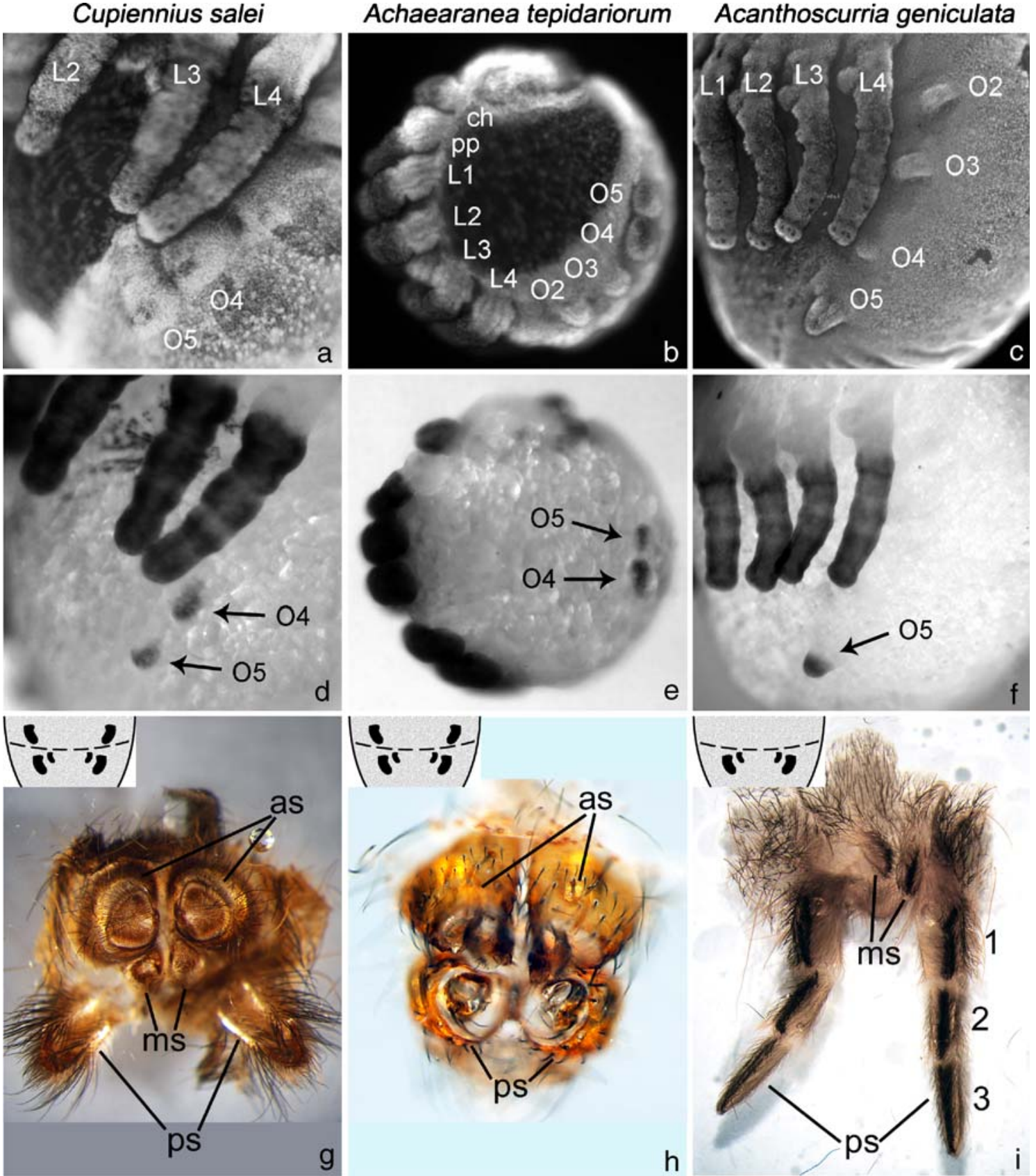

$\mathrm{h}$
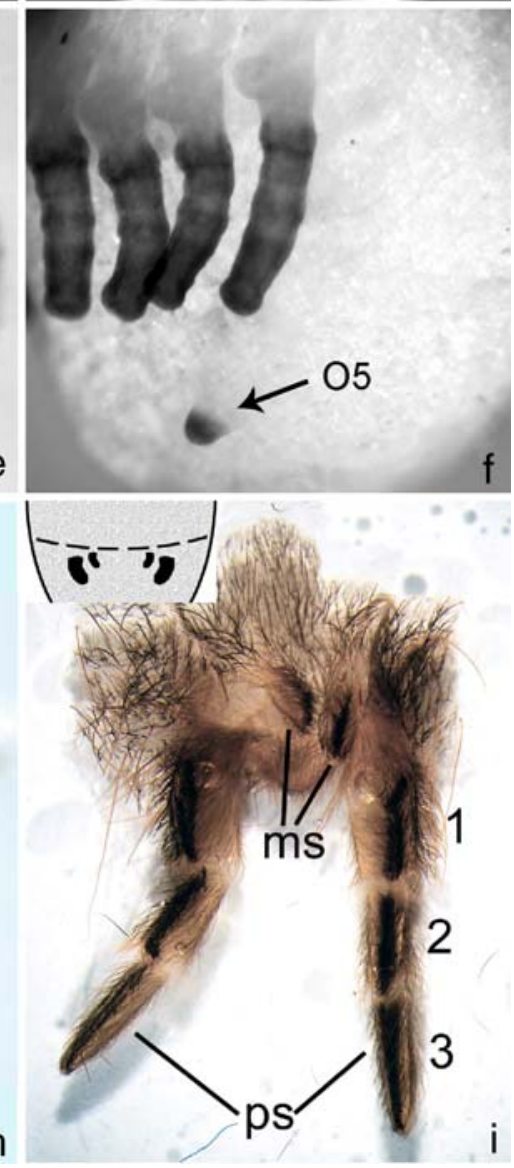

Fig. $8 \mathrm{Dll}$ expression prefigures the number of adult spinnerets. In $C$. salei $(\mathbf{a}, \mathbf{d}, \mathbf{g})$ and $A$. tepidariorum $(\mathbf{b}, \mathbf{e}, \mathbf{h})$ large spinneret primordia develop on opisthosomal segments 4 and $5(\mathbf{a}, \mathbf{b})$. Both primordia pairs express $D l l(\mathbf{d}, \mathbf{e})$ and will give rise to one anterior and one posterior pair of spinnerets in the adult $(\mathbf{g}, \mathbf{h})$. By contrast, in $A$. geniculata $(\mathbf{c}, \mathbf{f}, \mathbf{i})$, only the primordium in opisthosomal segment 5 is large (c), expresses Dll (f) and gives rise to a pair of posterior spinnerets in the adult (i). The embryos in a-c are Sytox Green stained. $\mathbf{g}-\mathbf{i}$ Cuticle preparations of the posterior end of the spider species including the spinnerets. Note the presence of median spinnerets in $\mathbf{g}$ and $\mathbf{i}$. These are also present in A. tepidariorum, but cannot be seen in the figure, because they are small and covered by the posterior spinnerets. Also note that the posterior spinnerets in $A$. geniculata are fully segmented (segments are denoted by numbers), and anterior spinnerets are lacking in the adult. The insets in $\mathbf{g - i}$ are schematic drawings of the opisthosomal segments 4 and 5 (denoted by the dotted line) showing the full adult complement of spinnerets, including the smaller accessory median spinnerets of opisthosomal segment 5. The drawings are simplified after Foelix (1996). Abbreviations see Figs. 2 and 3. Additional abbreviation: as anterior spinnerets, $m s$ median spinnerets, $p s$ posterior spinnerets 
legs, expression of $D l l$ prefigures the structures that will be retained in the adult, and lack of $D l l$ correlates with the degeneration of the structures in the embryo. This conclusion is further supported by the expression pattern of $D l l$ in the spinnerets of C. salei and A. tepidariorum. In both species (both belong to the Araneomorphae), anterior and posterior spinnerets are present in the adult (Fig. $8 \mathrm{~g}$, h), and in both species, Dll is expressed in both spinneret pairs during embryonic development (Fig. 8a, b, d, e). Thus, the expression of $D l l$ prefigures the number of adult spinnerets.

Acknowledgements We thank Marco Winkler for the technical assistance and the members of our laboratory for many helpful discussions. We thank Wim Damen for the critical reading and comments on the manuscript. This work has been funded by the German Research Council (DFG ENP grant PR1109/1-1).

Open Access This article is distributed under the terms of the Creative Commons Attribution Noncommercial License which permits any noncommercial use, distribution, and reproduction in any medium, provided the original author(s) and source are credited.

\section{References}

Abu-Shaar M, Mann RS (1998) Generation of multiple antagonistic domains along the proximodistal axis during Drosophila leg development. Development 125:3821-3830

Abzhanov A, Kaufman TC (2000) Homologs of Drosophila appendage genes in the patterning of arthropod limbs. Dev Biol 227:683-689

Beermann A, Jay DG, Beeman RW, Hülskamp M, Tautz D, Jürgens G (2001) The Short antennae gene of Tribolium is required for limb development and encodes the orthologue of the Drosophila Distal-less protein. Development 128:287-297

Damen WGM, Hausdorf M, Seyfarth EA, Tautz D (1998) A conserved mode of head segmentation in arthropods revealed by the expression pattern of Hox genes in a spider. Proc Natl Acad Sci USA 95:10665-10670

Damen WGM, Saridaki T, Averof M (2002) Diverse adaptations of an ancestral gill: a common evolutionary origin for wings, breathing organs, and spinnerets. Curr Biol 12:1711-1716

Dong PD, Chu J, Panganiban G (2000) Coexpression of the homeobox genes Distal-less and homothorax determines Drosophila antennal identity. Development 127:209-216

Dong PD, Dicks JS, Panganiban G (2002) Distal-less and homothorax regulate multiple targets to pattern the Drosophila antenna. Development 129:1967-1974

Foelix RF (1996) Biology of spiders, 2nd edn. Oxford University Press, New York

Khila A, Grbic M (2007) Gene silencing in the spider mite Tetranychus urticae: dsRNA and siRNA parental silencing of the Distal-less gene. Dev Genes Evol 217:241-251

Kimm MA, Prpic NM (2006) Formation of the arthropod labrum by fusion of paired and rotated limb-bud-like primordia. Zoomorphology 125:147-155

McGregor AP, Hilbrant M, Pechmann M, Schwager EE, Prpic NM, Damen WGM (2008) Cupiennius salei and Achaearanea tepidariorum: spider models for investigating evolution and development. BioEssays 30:487-498
Mittmann B, Scholtz G (2003) Development of the nervous system in the "head" of Limulus polyphemus (Chelicerata: Xiphosura): morphological evidence for a correspondence between the segments of the chelicerae and of the (first) antennae of Mandibulata. Dev Genes Evol 213:9-17

Prpic NM, Damen WGM (2004) Expression patterns of leg genes in the mouthparts of the spider Cupiennius salei (Chelicerata: Arachnida). Dev Genes Evol 214:296-302

Prpic NM, Damen WGM (2009) Notch-mediated segmentation of the appendages is a molecular phylotypic trait of the arthropods. Dev Biol 326:262-271

Prpic NM, Tautz D (2003) The expression of the proximodistal axis patterning genes Distal-less and dachshund in the appendages of Glomeris marginata (Myriapoda: Diplopoda) suggests a special role of these genes in patterning the head appendages. Dev Biol 260:97-112

Prpic NM, Telford MJ (2008) Expression of homothorax and extradenticle mRNA in the legs of the crustacean Parhyale hawaiensis: evidence for a reversal of gene expression regulation in the pancrustacean lineage. Dev Genes Evol 218:333-339

Prpic NM, Wigand B, Damen WGM, Klingler M (2001) Expression of dachshund in wild-type and Distal-less mutant Tribolium corroborates serial homologies in insect appendages. Dev Genes Evol 211:467-477

Prpic NM, Janssen R, Wigand B, Klingler M, Damen WGM (2003) Gene expression in spider appendages reveals reversal of exd/hth spatial specificity, altered leg gap gene dynamics, and suggests divergent distal morphogen signaling. Dev Biol 264:119-140

Prpic NM, Janssen R, Damen WGM, Tautz D (2005) Evolution of dorsalventral axis formation in arthropod appendages: H15 and optomotorblind/bifid-type T-box genes in the millipede Glomeris marginata (Myriapoda: Diplopoda). Evol Dev 7:51-57

Prpic NM, Schoppmeier M, Damen WGM (2008a) Collection and fixation of spider embryos. Cold Spring Harb Protoc. doi:10.1101/pdb.prot5067

Prpic NM, Schoppmeier M, Damen WGM (2008b) Whole-mount in situ hybridization of spider embryos. Cold Spring Harb Protoc. doi: $10.1101 /$ pdb.prot5068

Prpic NM, Schoppmeier M, Damen WGM (2008c) Dissecting spider embryos for light microscopy. Cold Spring Harb Protoc. doi: $10.1101 /$ pdb.prot5072

Ramsköld L, Edgecombe GD (1996) Trilobite appendage structure Eoredlichia reconsidered. Alcheringa 20:269-276

Ronco M, Uda T, Mito T, Minelli A, Noji S, Klingler M (2008) Antenna and all gnathal appendages are similarly transformed by homothorax knock-down in the cricket Gryllus bimaculatus. Dev Biol 313:80-92

Schoppmeier M, Damen WGM (2001) Double-stranded RNA interference in the spider Cupiennius salei: the role of Distalless is evolutionarily conserved in arthropod appendage formation. Dev Genes Evol 211:76-82

Stollewerk A (2002) Recruitment of cell groups through Delta/Notch signalling during spider neurogenesis. Development 129:53395348

Telford MJ, Thomas RH (1998) Expression of homeobox genes shows chelicerate arthropods retain their deutocerebral segment. Proc Natl Acad Sci USA 95:10671-10675

Toegel JP, Wimmer EA, Prpic NM (2009) Loss of spineless function transforms the Tribolium antenna into a thoracic leg with pretarsal, tibiotarsal and femoral identity. Dev Genes Evol 219:53-58

Waloßek D (1995) The Upper Cambrian Rehbachiella, its larval development, morphology and significance for the phylogeny of Branchiopoda and Crustacea. Hydrobiologia 298:1-13 